\title{
Cavity-enhanced light-matter interaction in Vogel-spiral devices as a platform for quantum photonics
}

Oliver J. Trojak, ${ }^{1, *}$ Sean Gorsky, ${ }^{2,}$ Connor Murray, ${ }^{1}$ Fabrizio Sgrignuoli, ${ }^{2}$

Felipe A. Pinheiro, ${ }^{3}$ Luca Dal Negro, $2,4,5, \dagger$ and Luca Sapienza $\$ 1$

${ }^{1}$ Department of Physics and Astronomy, University of Southampton, Southampton SO17 1BJ, United Kingdom

${ }^{2}$ Department of Electrical \& Computer Engineering and Photonics Center,

Boston University, 8 Saint Mary's St., Boston, Massachusetts, 02215, USA.

${ }^{3}$ Instituto de Fisica, Universidade Federal do Rio de Janeiro, Rio de Janeiro-RJ 21941-972, Brazil

${ }^{4}$ Division of Material Science \& Engineering, Boston University,

15 Saint Mary's St. Brookline, Massachusetts, 02446, USA

${ }^{5}$ Department of Physics, Boston University,

590 Commonwealth Ave., Boston, Massachusetts, 02215, USA

\footnotetext{
\$ Author to whom correspondence should be addressed: 1. sapienza@soton.ac.uk

Website: www. quantum. soton.ac.uk
} 


\begin{abstract}
Enhancing light-matter interactions on a chip is of paramount importance for classical and quantum photonics, sensing and energy harvesting applications. Several photonic geometries have been developed, allowing high extraction efficiencies, enhanced light-matter interactions and control over the spontaneous emission dynamics of solid-state quantum light sources. To this end, a device geometry resilient to nanofabrication imperfections, providing high-quality light confinement and control over the emitted light properties, would be desirable. We demonstrate that aperiodic arrangements, whose geometry is inspired by natural systems where scattering elements are arranged following Fibonacci series, represent a platform for enhancing the light-matter interaction in on-chip nano-photonic devices, allowing to achieve efficient visible light confinement. We use optically-active defect centers in silicon nitride as internal light sources to image and characterize, by means of micro-photoluminescence spectroscopy, the individual optical modes confined by photonic membranes with Vogel-spiral geometry. By studying the statistics of the measured optical resonances, in partnership with rigorous multiple scattering theory, we observe log-normal distributions and report quality factors with values as high as $2201 \pm 443$. Our findings improve the understanding of the fundamental physical properties of light-emitting Vogel-spiral systems and show their application to active nano-photonic devices. These results set the basis for further development of quantum devices that leverage the unique properties of aperiodic Vogel spiral order on a chip, including angular momentum states, thus producing mode structures for information processing and communications on the chip.
\end{abstract}


Engineered photonic devices play a key role in the development of on-chip sources of classical and quantum light for a variety of applications including nano-lasers ${ }^{1}$, single-photon sources ${ }^{2}$, sensors $^{3}$, and energy harvesters ${ }^{4}$. Ideally, one would like nano-photonic device geometries that show: scalability (i.e., the ability of fabricating more than one device that has exactly the same, as-designed, optical properties), enhanced light-matter interaction (tunable to a broad range of wavelengths, from the visible to the telecommunication band), control over spontaneous emission dynamics (allowing, for instance, enhanced coherence in the emission and increased repetition rates) and control over the properties of the emitted light (such as the emission directionality and its optical angular momentum). Different photonic geometries have been developed to achieve these goals and, among those, photonic crystals have showed high quality in light confinement both at telecommunications and near-infrared wavelength ${ }^{5}$, and strong lifetime control ${ }^{6}$; however scalability and reproducibility of the results is an issue, since nanometer-scale accuracy in the fabrication is needed ${ }^{7}$. This is particularly evident for devices operating at visible wavelengths where, given the dimensions involved, two-dimensional photonic crystal cavities have proved to be much more challenging to fabricate compared to those operating at longer wavelengths, resulting in limited performances that further depart from simulated values ${ }^{8,9}$. Micro-pillar geometries have assured high-brightness and coherent emission ${ }^{10}$ and planar gratings have allowed the realisation of broadband, bright and pure single-photon emission ${ }^{11}$. On the other hand, disordered photonic crystal waveguides have allowed less stringent fabrication requirements, high-quality light confinement over a wide range of wavelengths ${ }^{12,13}$, control over the spontaneous emission dynamics of single emitters ${ }^{14}$ and optical sensing ${ }^{15}$. Control over the optical angular momentum has also been achieved by integrating emitters within metamaterials ${ }^{16}$.

In this work, we propose a photonic platform, based on aperiodic geometries, that allows efficient visible light confinement and the co-existence of several confined optical modes within devices that require less stringent fabrication accuracies, compatible with silicon technology and with the integration of quantum emitters operating at visible wavelengths, like defect centers in diamond, two-dimensional materials and molecules.

Such a design relies on the concept of aperiodic order ${ }^{17-19}$ that, for instance, characterizes the peculiar geometry of quasi-crystals ${ }^{20,21}$, i.e. long-range ordered systems that lack translational symmetry, to achieve light confinement in two and three dimensions. Beyond quasicrystals, aperiodic order can be used to engineer photonic devices with well-defined deterministic mathematical rules $^{18,22,23}$, providing compatibility with planar nano-fabrication technologies ${ }^{24}$, as well as dis- 
tinctive spectral and optical properties ${ }^{25-28}$, including optical angular momentum ${ }^{29}$, characteristics that make them appealing for quantum photonics applications ${ }^{30-33}$.

We demonstrate the potential of nano-photonic devices characterised by a bio-inspired deterministic aperiodic structure: in particular, we design, fabricate, and characterize bio-inspired nano-devices with Vogel-spiral geometry (see Fig. 1(a) and Supplementary Information for more details) that were originally introduced in relation to the fascinating geometrical problems of phyllotaxis ${ }^{34-36}$. This particular deterministic aperiodic structure was intensively studied in the last decade, and was found to have interesting fundamental optical properties for nano-photonic and nano-plasmonic applications, including: vector-wave localization ${ }^{37}$, polarization-insensitive light diffraction ${ }^{38}$, enhanced second-harmonic generation, increased light extraction ${ }^{31,39,40}$, control of spontaneous emission dynamics ${ }^{41}$, and omni-directional photonic band gaps ${ }^{42-44}$. In this work, we show that Vogel spiral active membranes enable to achieve efficient light confinement and represent a platform for enhancing the light-matter interaction on a chip.

We experimentally investigate, by means of micro-photoluminescence spectroscopy, the optical properties of suspended silicon nitride $\left(\mathrm{Si}_{3} \mathrm{~N}_{4}\right)$ membranes, where air holes are arranged in a golden-angle Vogel-spiral geometry (see Fig. 1(a) and Supplementary Information). The devices are designed by evaluating the local density of optical states (LDOS) computed with an accurate spectral method. The LDOS describes the radiation dynamics of a source embedded into an arbitrary structure, from which the spectral location of high quality factor modes, such as those near the band-gap $28,43,45,46$, can be predicted. Specifically, we utilized the rigorous theory of multipolar expansion to evaluate the two-dimensional electromagnetic Green's tensor, from which the LDOS is calculated (for more details, see Supplementary Information). The LDOS is evaluated for arbitrary arrays of parallel and non-overlapping circular cylinders embedded in a non-absorbing medium. The analysis is limited to the transverse electric polarization only, as this is the polarization for which a band-gap is expected to occur in an air-hole membrane ${ }^{47}$. To take into account the out-of-plane losses, we have applied an effective refractive index method where the material dispersion of the dielectric material is replaced by the effective index of the fundamental guided mode in the unperturbed (without air-holes) three-dimensional heterostructure ${ }^{48}$ (see Supplementary Informations for more details). Fig. 1(b) shows a map of the LDOS as a function of wavelength and air-hole diameters $d$ etched in a silicon nitride membrane, and (c) depicts onedimensional cuts. Specifically, the LDOS is evaluated at the center of a spiral characterized by 350 air-holes and by an averaged inter-particle separation of $273 \mathrm{~nm}$. Clear photonic band-gap and 
pseudo-gaps (secondary gaps of smaller amplitudes), identified by bright yellow streaks in Fig. 1 (b), are visible and their behaviour is determined by the unique multi-fractal structural properties of the golden-angle Vogel spiral ${ }^{28}$. For example, there are several peaks, more clearly visible in Fig. 1 (c), which correspond to long-lived modes generated by the first-neighbor distributions of the spiral elements ${ }^{37}$. Localized band-edge modes are formed when ring-shaped regions with similar hole separation are sandwiched between two regions with different hole separations, creating a photonic heterostructure ${ }^{28}$. Moreover, these band-edge modes are often spatially extended, longlived $^{37}$, and less sensitive to local perturbations. This makes golden-angle Vogel spirals a very appealing photonic platform, due to more robust fabrication tolerances than traditional photonic crystals $^{42}$. The presence of band-gaps despite the relatively low index contrast between silicon nitride and air is related to the long-range order in a nearly-isotropic geometry ${ }^{22,28,43,49}$. Isotropic gaps also imply reduced group velocity modes and therefore increased light-matter interaction, thus making these devices interesting for non-linear optics applications and for the realisation of low-threshold lasers ${ }^{42,47}$.

We fabricate free-standing $340 \mathrm{~nm}$-thick membranes in silicon nitride grown by plasmaenhanced chemical vapour deposition on a silicon substrate, into which 1000 air holes arranged in a golden-angle spiral geometry are etched (see the Supplementary Information, for more details about the growth and the fabrication of the devices). A scanning electron micrograph image of a fabricated device is shown in Fig. 1(a). As previously reported, $\mathrm{Si}_{3} \mathrm{~N}_{4}$ can emit radiation over a broad range of wavelengths, typically spanning from $\sim 600$ to $\sim 850 \mathrm{~nm}$, once excited with external light sources ${ }^{13}$. We will use such intrinsic photo-luminescence as the internal light source to characterize the confined optical modes ${ }^{13}$ supported by the golden-angle spiral structure.

The fabricated sample is placed under a confocal optical microscope and illuminated with a $455 \mathrm{~nm}$-light emitting diode that excites the silicon nitride luminescence over an area of about $50 \times 50 \mu \mathrm{m}^{2}$. The emission is then imaged on an electron-multiplied charge-coupled device (see Fig. 2(a)). This set-up allows us to image the confined optical modes, as shown in Fig. 2(b) where a photo-luminescence image of the optical modes with wavelengths above $550 \mathrm{~nm}$ confined by the structure is superimposed to the SEM of the fabricated device. We observe a high-intensity region in the center and at the border of the device and confined modes in the photonic crystal area.

The sample is then excited with a $405 \mathrm{~nm}$ continuous-wave laser with an excitation spot diameter of $\sim 2 \mu \mathrm{m}$, which allows light excitation and collection from specific areas of the nano-photonic devices. The emitted light is collected and sent to a grating spectrometer equipped with a charge- 
coupled device for spectral characterization (see Fig. 2(a)).

An example of a collected micro-photoluminescence spectrum is shown in Fig. 3(a), where sharp resonances, a signature of light confinement, appear above the broad emission from the silicon nitride material. By fitting the resonant peaks with Lorentzian functions, we extract quality factors reaching $2201 \pm 443$ (see Fig. 3(b,c)), thus exceeding values reported for photonic crystal cavities in silicon nitride operating at visible wavelengths ${ }^{8,9}$.

The optical resonances supported by open and planar Vogel spirals are embedded in a threedimensional environment and they can leak out of the two-dimensional plane, according to their quality factors. As a result light is not fully confined in the spiral plane and the resulting optical resonances are actually three-dimensional electromagnetic quasi-modes ${ }^{37}$. The aperiodic photonic devices under study provide a large number of optical resonances, resembling the behaviour observed in disordered photonic crystal waveguides, confining light along one dimension in the plane $^{13}$. However, the resonances under study are three-dimensional electromagnetic quasi-modes with two-dimensional geometrical support of Vogel spiral arrays, with clear advantages for applications since the higher dimensionality of the present devices provides more easily addressable and larger active areas.

In Ref. ${ }^{41}$, it was demonstrated that the lifetime of single quantum dots operating at nearinfrared wavelength is strongly modified by the modulation of the density of optical states due to the aperiodic photonic structure. In that work, the spectral signature of confined optical modes was not clearly visible in the photoluminescence spectra. In the present work, instead, by using the broad and homogeneous intrinsic photoluminescence of silicon nitride at visible wavelengths ${ }^{13}$, we probe the confined optical modes and analyse their wavelength and quality factor distributions as a function of the structural parameters of the photonic devices under investigation.

We have extensively characterized and tested $\mathrm{Si}_{3} \mathrm{~N}_{4}$ devices with 165, 190, and $215 \mathrm{~nm}$ diameter of the air holes. Examples of the statistics of the collected optical resonances, plotted as a function of wavelength and quality factor $Q$ (a factor that estimates the quality in the light confinement, evaluated as the ratio between the optical resonance central wavelength and its linewidth), are shown in Fig. 4. We observe that reducing the air-hole diameter (while keeping the rest of the parameters constant) results in a shift of the optical resonances towards longer wavelengths, in agreement with the band-gap calculations shown in Fig.1(c), and in an increase in the average quality factors (see Supplementary Information for more discussion, including a comparison to simulations). We also show a wide tunability of the wavelength of the confined optical modes over 
a $100 \mathrm{~nm}$ wavelength window (see Fig. 4(a)), proving the suitability of aperiodic systems for the realisation of devices with broad-band operation. Concurrently, reducing the air-hole diameters yields an increase in quality factors. This trend is opposite to what is predicted in our simulations, which are only able to predict in-plane quality factors, neglecting the increased out-of-plane losses of more strongly confined modes ${ }^{50}$ (more details can be found in the Supplementary Informations).

Fig. 4(b) shows that the probability distributions of the measured quality factors follow lognormal statistics. Interestingly, the log-normal distribution of $Q$-factors has been predicted and observed in disordered systems in the Anderson-localized regime ${ }^{51-53}$. However, differently from traditional random media where the log-normal distribution of $Q$-factors is associated to exponentially localized modes, in Vogel spirals this behaviour is related to the multi-length scale decay of optical resonances, which reflects the multi-fractal complexity of the Vogel-spiral geometry and its $\operatorname{LDOS}^{28}$ (see Supplementary Informations for more details).

The quality factor values are limited by the relatively small index contrast between silicon nitride and air and we expect that higher values could be obtained using other materials and/or operating at longer wavelengths. In Ref. ${ }^{41}$, it was shown that the light-matter enhancement allowed one to reach the weak coupling regime where the recombination mechanisms of the excitations is an irreversible process whose temporal dynamics can be modified. The achievement of the light-matter strong coupling regime, where the system cycles between the excited and the ground state of the photonic and matter excitations, would require small mode volumes that might not be provided by all the confined optical modes in the structure under study, since some of them are spatially extended (see Fig. 2(b) for examples of far-field distributions of some of the modes sustained by the aperiodic devices).

In conclusion, Our results show that nano-photonic devices based on the bio-inspired Vogel spiral geometry provide an effective platform for efficient light confinement on a chip, particularly important in nano- and quantum photonics given the availability of high-quality visible quantum emitters, including atoms, defect centers (in diamond, silicon carbide and two-dimensional materials), molecules, droplet and colloidal quantum dots ${ }^{2}$ and for cavity quantum electrodynamics experiments with quantum emitters ${ }^{41}$ in the visible range of wavelengths. We foresee the application of engineered aperiodic geometries for integrated quantum photonics, nano-lasers, optical sensors and non-linear optics ${ }^{17}$ and the development of active devices operating from the visible to the telecommunication range of wavelengths. In particular, the efficient light confinement that we have demonstrated in Vogel spiral structures could be utilized for the development of single-photon 
devices where optical angular momentum is imparted to the emitted radiation and used as an extra degree of freedom to encode information for quantum information technology applications on a $\operatorname{chip}^{29,38,39,54}$.

\section{Supplementary Material}

Supplementary material provides more in-depth discussion of the device geometry, fabrication details and simulations.

\section{Data Availability Statement}

The data that support the findings of this study are available from the corresponding author upon reasonable request.

\section{Acknowledgments}

FAP acknowledges financial support from CNPq, CAPES, and FAPERJ. LDN acknowledges partial support from the Army Research Laboratory under Cooperative Agreement Number W911NF-12-2-0023 for the development of theoretical methods utilized in the paper. LS acknowledges partial support from the Royal Society, grant RG170217, the Leverhulme Trust, grant IAF-2019-013, EPSRC, grant EP/P001343/1.

\section{Author contributions}

LS conceived the optical set-up and built it together with OJT. OJT grew the silicon nitride material, fabricated the devices, carried out the experiments and analysed the data, together with LS. CM contributed to the optical characterisation and data analysis. LS, FAP, LDN conceived the research activities and discussed the results, together with the other authors. LS supervised the experimental part of the project and wrote the manuscript with contributions from the other authors. LDN supervised the design and modeling contributions. SG developed the numerical tools utilized in the paper. SG and FS performed, analyzed, and organized the simulation results. 
SG wrote the Supplementary Informations with the help of FS and LDN.

* These authors contributed equally to this work

$\dagger$ Electronic address: dalnegro@bu.edu

1 R.-M. Ma, R.F. Oulton, Applications of nanolasers, Nature Nanotechnology 14, 12 (2019).

2 I. Aharonovich, D. Englund, M. Toth, Solid-state single-photon emitters, Nat. Photonics 10, 631 (2016).

3 J. Hodgkinson, R.P. Tatam, Optical gas sensing: a review, Meas. Sci. Technol. 24, 012004 (2013).

4 B. Parida, S. Iniyan, R. Goic, A review of solar photovoltaic technologies. Renewable Sustainable Energy Rev. 15, 1625 (2011).

5 T. Asano, Y. Ochi, Y. Takahashi, K. Kishimoto, S. Noda, Photonic crystal nanocavity with a Q factor exceeding eleven million, Optics Express 25, 1769 (2017).

6 T.W. Ebbesen, Hybrid Light-Matter States in a Molecular and Material Science Perspective, Acc. Chem. Res. 49, 2403 (2016).

7 Y. Akahane, T. Asano, B.-S.Song, S. Noda, High-Q photonic nanocavity in a two-dimensional photonic crystal, Nature 425, 944 (2003).

8 M. Makarova, J. Vuckovic, H. Sanda, Y. Nishi, Silicon-based photonic crystal nanocavity light emitters, Appl. Phys. Lett. 89, 221101 (2006).

9 M. Barth, J. Kouba, J. Stingl, B. Lochel, O. Benson, Modification of visible spontaneous emission with silicon nitride photonic crystal nanocavities, Opt. Express 15, 17231 (2007).

10 N. Somaschi, V. Giesz, L. De Santis, J. C. Loredo, M. P. Almeida, G. Hornecker, S. L. Portalupi, T. Grange, C. Antn, J. Demory, C. Gomez, et al., Near-optimal single-photon sources in the solid state, Nature Photonics 10, 340 (2016).

11 L. Sapienza, M. Davanco, A. Badolato, K. Srinivasan, Nanoscale optical positioning of single quantum dots for bright and pure single-photon emission, Nature Communications 6, 7833 (2015).

12 J. Topolancik, B. Ilic, F. Vollmer, Experimental observation of strong photon localization in disordered photonic crystal waveguides, Phys. Rev. Lett. 99, 253901 (2007).

13 T. Crane, O.J. Trojak, J.P. Vasco, S. Hughes, L. Sapienza, Anderson localisation of visible light on a nanophotonic chip, ACS Photonics 4, 2274 (2017).

14 L. Sapienza, H. Thyrrestrup, S. Stobbe, P.D. Garcia, S. Smolka, P. Lodahl, Cavity quantum electrodynamics with Anderson-localized modes, Science 327, 1352 (2010). 
15 O.J. Trojak, T. Crane, L. Sapienza, Optical sensing with Anderson-localised light, Applied Physics Letters 111, 141103 (2017).

16 Y. Kan, S.K.H. Andersen, F. Ding, S. Kumar, C. Zhao, S.I. Bozhevolnyi, Metasurface-enabled generation of circularly polarized single photons, Adv. Mater. 32, 1907832 (2020).

17 L. Dal Negro, S.V. Boriskina, Deterministic aperiodic nanostructures for photonics and plasmonics applications, Laser \& Photonics Reviews 6, 178 (2012).

18 Z.V.Vardeny, A. Nahata, A. Agrawal, Optics of photonic quasicrystals, Nature Photonics 7, 177 (2013).

19 M. Baake, U. Grimm, Aperiodic order, Cambridge University Press (2013).

20 C. Janot, Quasicrystals A Primer, Clarendon Press, Oxford, (1992).

21 D. Levine, P.J. Steinhardt, Quasicrystals: a new class of ordered structures, Physical review letters 53, 2477 (1984).

${ }^{22}$ L. Dal Negro, Y. Chen, F. Sgrignuoli, Aperiodic photonics of elliptic curves, Crystals 9, 482 (2019).

23 L. Dal Negro, R. Wang, F. Pinheiro, Structural and spectral properties of deterministic aperiodic optical structures, Crystals 6, 161 (2016).

24 L. Dal Negro, Optics of aperiodic structures: fundamentals and device applications, CRC Press (2013).

25 L. Dal Negro, S. Inampudi, Fractional transport of photons in deterministic aperiodic structures, Scientific Reports 7, 2259 (2017).

26 P. Barthelemy, J. Bertolotti, D.S. Wiersma, A Lévy flight for light, Nature 453, 495 (2008).

27 W. Gellerman, M. Kohmoto, B. Sutherland, P.C. Taylor, Localization of light waves in Fibonacci dielectric multilayers, Physical Review Letters 72, 633 (1994).

28 J. Trevino, S.F. Liew, H. Noh, H. Cao, L. Dal Negro, Geometrical structure, multifractal spectra and localized optical modes of aperiodic Vogel spirals, Optics Express 20, 3015 (2012).

29 L. Dal Negro, N. Lawrence, J. Trevino, Analytical light scattering and orbital angular momentum spectra of arbitrary Vogel spirals, Optics Express 20, 18209 (2012).

30 L. Mahler, A. Tredicucci, F. Beltram, C. Walther, J. Faist, H.E. Beere, D.A. Ritchie, D.S. Wiersma , Quasi-periodic distributed feedback laser, Nature Photonics 4, 165 (2010).

31 K. Guo, M. Du, C.I. Osorio, A.F. Koenderink, Broadband light scattering and photoluminescence enhancement from plasmonic Vogel's golden spirals, Laser \& Photonics Reviews 11, 1600235 (2017).

32 M. Notomi, H. Suzuki, T. Tamamura, K. Edagawa, Lasing action due to the two-dimensional quasiperiodicity of photonic quasicrystals with a Penrose lattice, Physical Review Letters 92, 123906 (2004).

33 E. Macia, The role of aperiodic order in science and technology, Reports on Progress in Physics 69, 397 
(2005).

34 G.J. Mitchison, Phyllotaxis and the Fibonacci series, Science 196, 270 (1977).

35 R.V. Jean, Phyllotaxis. A systemic study in plant morphogenesis, Cambridge University Press, New York, USA (1994).

36 M. Naylor, Golden and $\pi$ flowers: A spiral story, Mathematics Magazine 75, 163 (2002).

37 F. Sgrignuoli, R. Wang, F.A. Pinheiro, L. Dal Negro, Localization of scattering resonances in aperiodic Vogel spirals, Physical Review B 99, 104202 (2019).

38 J. Trevino, H. Cao, L. Dal Negro, Circularly symmetric light scattering from nanoplasmonic spirals, Nano Letters 11, 2008 (2011).

39 N. Lawrence, J. Trevino, L. Dal Negro, Control of optical orbital angular momentum by Vogel spiral arrays of metallic nanoparticles, Optics Letters 37, 5076 (2012).

40 S. Gorsky, R. Zhang, A. Gok, R. Wang, K. Kebede, A. Lenef, M. Raukas, L. Dal Negro, Directional light emission enhancement from LED-phosphor converters using dielectric Vogel spiral arrays, APL Photonics 3, 126103 (2018).

41 O.J. Trojak, S. Gorsky, F. Sgrignuoli, F.A. Pinheiro, S.-I. Park, J.D. Song, L. Dal Negro, L. Sapienza, Cavity quantum electro-dynamics with solid-state emitters in aperiodic nano-photonic spiral devices, Applied Physics Letters 117, 124006 (2020).

42 M.E. Pollard, G.J. Parker, Low-contrast bandgaps of a planar parabolic spiral lattice, Optics Letters 34, 2805 (2009).

43 S.F. Liew, H. Noh, J. Trevino, L. Dal Negro, H. Cao, Localized photonic band edge modes and orbital angular momenta of light in a golden-angle spiral, Optics Express 19, 23631 (2011).

44 A. Agrawal, N. Kejalakshmy, J. Chen, B.M.A. Rahman, K.T.V. Grattan, Golden spiral photonic crystal fiber: polarization and dispersion properties, Optics Letters 33, 2716 (2008).

45 F. Sgrignuoli, G. Mazzamuto, N. Caselli, F. Intonti, F.S. Cataliotti, M. Gurioli, C. Toninelli, Necklace state hallmark in disordered 2D photonic systems, ACS Photonics 2, 1636 (2015).

46 S. John, Strong localization of photons in certain disordered dielectric superlattices, Physical Review Letters 58, 2486 (1987).

47 M. Soljačić, J.D. Joannopoulos, Enhancement of nonlinear effects using photonic crystals, Nature Materials 3, 211 (2004).

48 M. Qiu, Effective index method for heterostructure-slab-waveguide-based two-dimensional photonic crystals, Applied Physics Letters 81, 1163 (2002). 
49 M.C. Rechtsman, H.-C. Jeong, P.M. Chaikiin, S. Torquato, P.J. Steinhardt, Optimized structures for photonic quasicrystals, Physical Review Letters 101, 073902 (2008).

50 S.V. Boriskina, A. Gopinath, and L. Dal Negro, Optical gap formation and localization properties of optical modes in deterministic aperiodic photonic structures, Opt. Express 16, 18813 (2008).

51 F.A. Pinheiro, Statistics of quality factors in three-dimensional disordered magneto-optical systems and its applications to random lasers, Physical Review A 78, 023812 (2008).

52 S. Smolka, H. Thyrrestrup, L. Sapienza, T.B. Lehmann, K.R. Rix, L.S. Froufe-Perez, P.D. Garcia, P. Lodahl, Probing the statistical properties of Anderson localization with quantum emitters, New Journal of Physics 13, 063044 (2011).

53 M. Weiss, J.A. Méndez-Bermúdez, T. Kottos, Resonance width distribution for high-dimensional random media, Physical Review B 73, 045103 (2006).

54 G. Molina-Terriza, J.P. Torres, L. Torner, Twisted Photons, Nature Physics 3, 305 (2007). 
Figures 

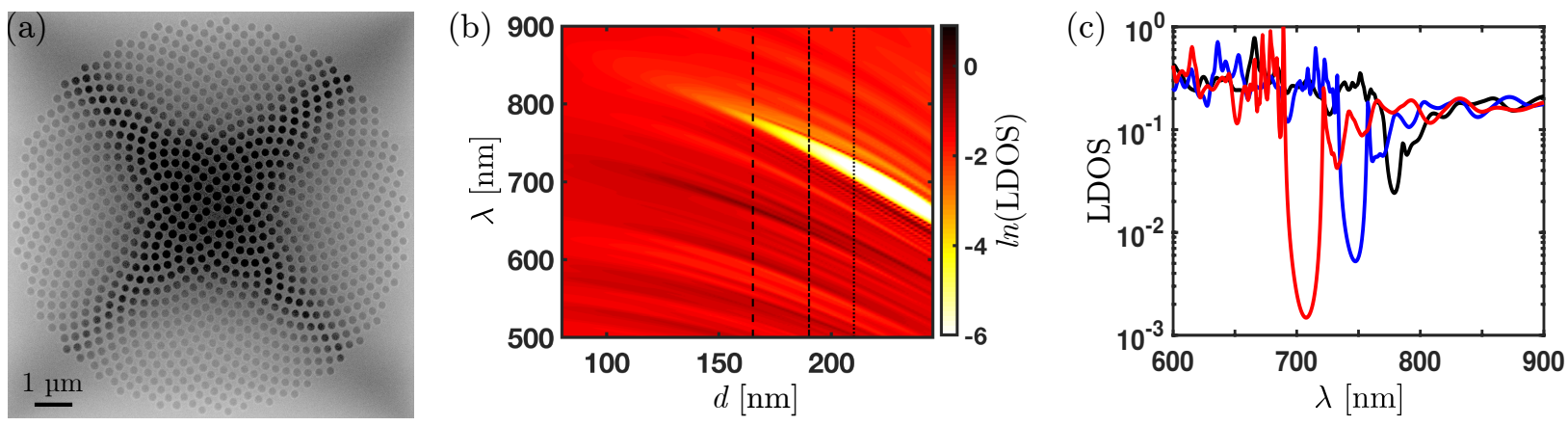

FIG. 1: $\mathrm{Si}_{3} \mathrm{~N}_{4}$ golden-angle Vogel-spiral device designs. (a) Scanning electron micrograph (SEM) of a suspended silicon nitride aperiodic photonic device with golden-angle Vogel-geometry. (b) Local density of optical states (LDOS) map calculated at the center of the spiral as a function of the wavelength $\lambda$ and of the air-hole diameters $d$. We have considered 101 different values of the $d$ parameter to design these structures. (c) One dimensional-LDOS line-cuts as a function of wavelength for three different hole diameters: 165 (black line), 190 (blue line), and $215 \mathrm{~nm}$ (red line), for devices with an average inter-particle separation of $273 \mathrm{~nm}$. 
(a)
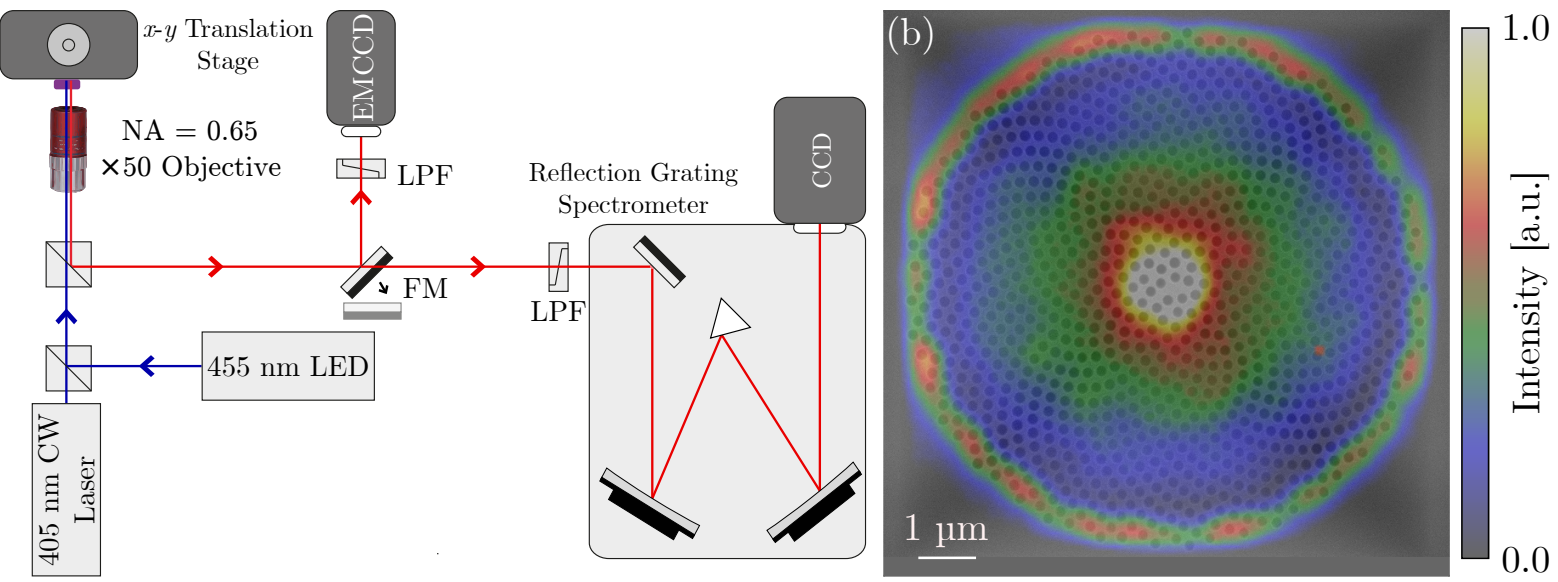

FIG. 2: Characterisation micro-photoluminescence set-up and optical imaging of the confined optical modes. (a) Schematic of the confocal micro-photoluminescence set-up (not to scale), comprising a light emitting diode (LED) with emission wavelength centred at $455 \mathrm{~nm}$, a continuous wave (CW) laser emitting at $405 \mathrm{~nm}$ as excitation source, focused by a $50 \times$ microscope objective (with numerical aperture NA $=0.65$ ) onto a sample placed on an $x y$-translation stage. The detection is carried out by an Electron Multiplying Charge Coupled Device (EMCCD) for photo-luminescence imaging, or by a CCD for spectral characterisation. $(\mathrm{LPF}=550 \mathrm{~nm}$ long-pass filter, $\mathrm{FM}=$ flip mirror), the squares represent beam-splitters. (b) Photoluminescence image, collected under 455-nm light-emitting diode illumination with a power density of $40 \mathrm{~W} / \mathrm{cm}^{2}$, of the optical modes with wavelengths above $550 \mathrm{~nm}$ confined by the structure is superimposed to the SEM of the fabricated device. 
(a)

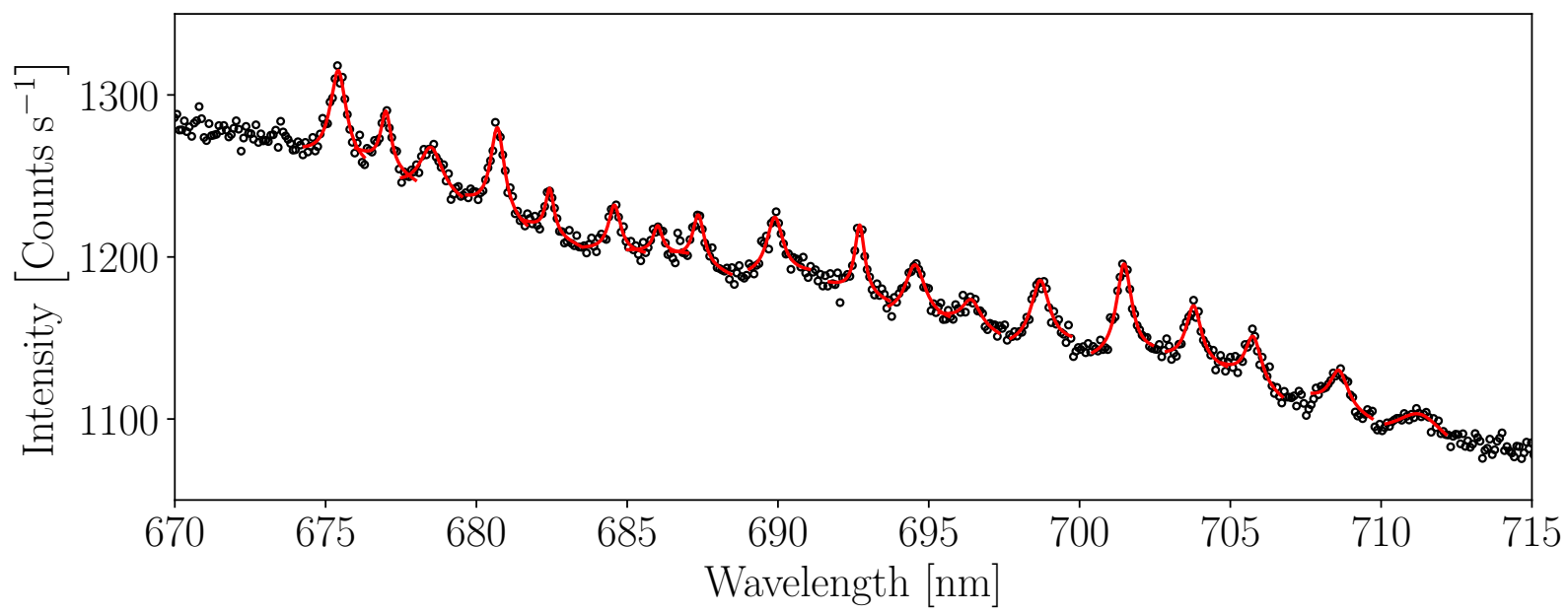

(b)

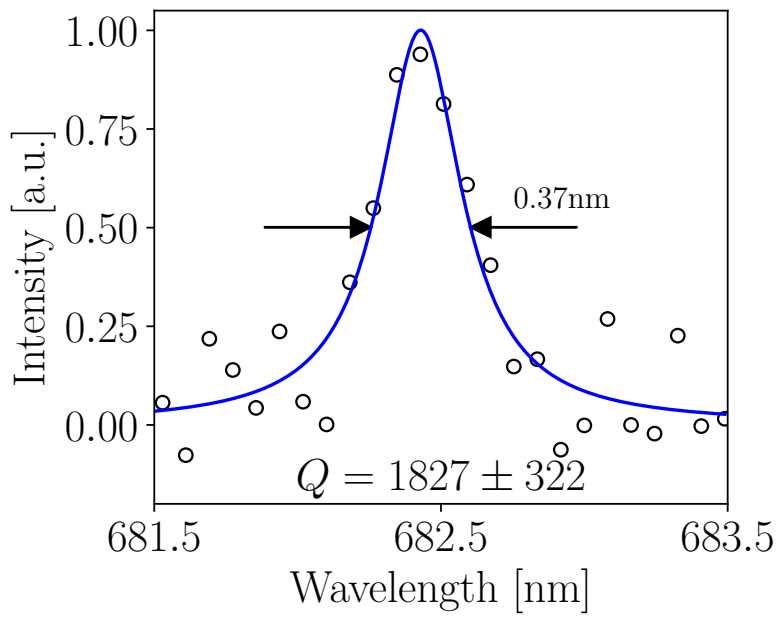

(c)

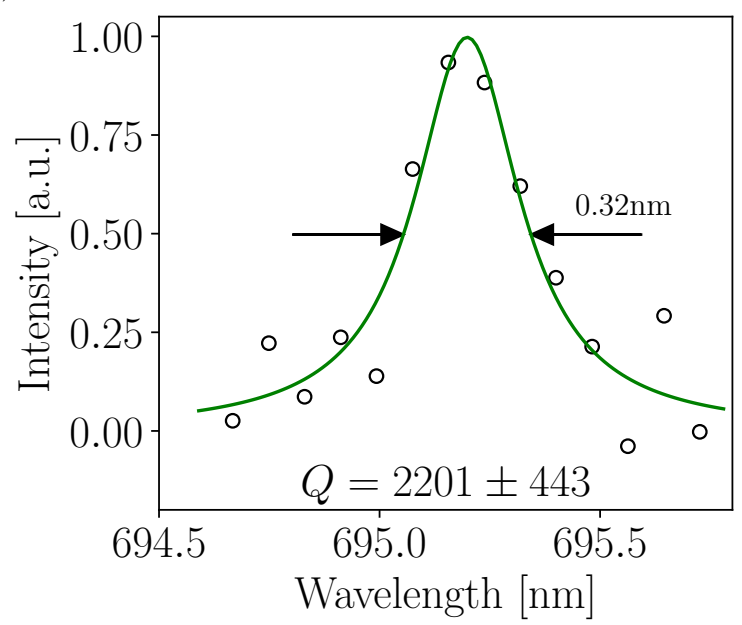

FIG. 3: Micro-photoluminescence measurements proving efficient light confinement on a $\mathrm{Si}_{3} \mathrm{~N}_{4}$ chip. (a) Example of a broad-range photo-luminescence spectrum showing sharp optical resonances, signature of light confinement in deterministic aperiodic photonic devices in silicon nitride. The spectrum was collected at room temperature, under $405 \mathrm{~nm} \mathrm{CW}$ laser illumination, with a power density of $1.8 \mathrm{~kW} / \mathrm{cm}^{2}$. The solid lines represent Lorentzian fits to the data (symbols). (b,c) Examples of optical resonances, collected under the same conditions as panel (a), showing Lorentzian fits and the extracted quality factors $Q$. 

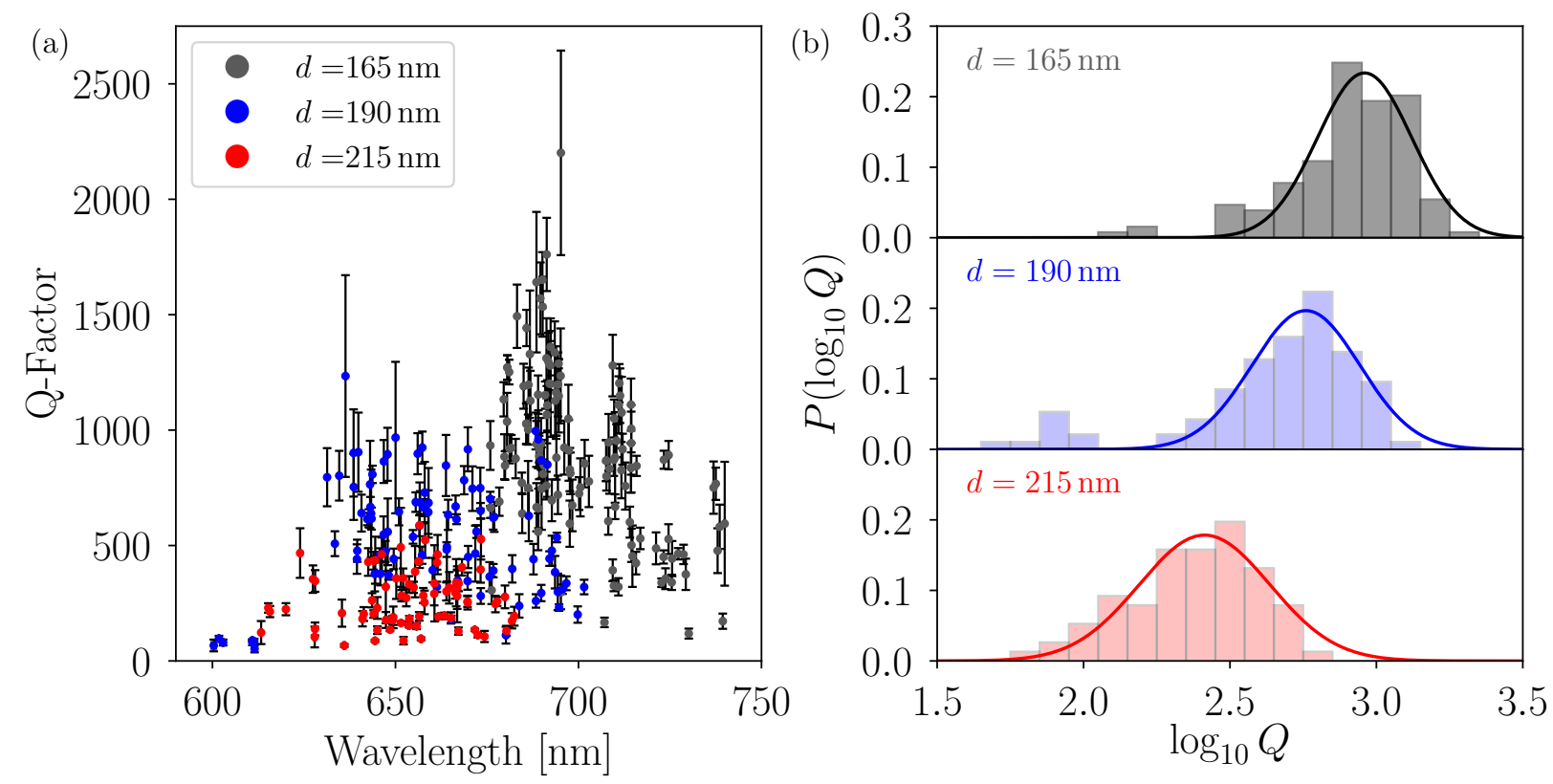

FIG. 4: Statistical analysis of the optical resonances confined by aperiodic $\mathrm{Si}_{3} \mathrm{~N}_{4}$ photonic devices. (a) Statistics of the quality factors distributions collected from micro-photoluminescence spectra, like the ones shown in Fig. 3, plotted as a function of emission wavelength, for aperiodic photonic devices with hole diameter of 165 (grey), 190 (blue), 215 (red) nm. (b) Probability distribution of the quality factors shown in panel (a), plotted with the same colour coding. Coloured continuous lines are log-normal fits to the data. 